\title{
Helicobacter pylori isolates from Greek children express type 2 and type 1 Lewis and $\alpha 1,6$-glucan antigens in conjunction with a functional type IV secretion system
}

\author{
Correspondence \\ Eleonora Altman \\ Eleonora.altman@nrc-cnrc.gc.ca \\ Dionyssios N. Sgouras \\ sgouras@pasteur.gr
}

Received 26 September 2011

Accepted 5 December 2011

\author{
Eleonora Altman, ${ }^{1}$ Vandana Chandan, ${ }^{1}$ Blair A. Harrison, ${ }^{1}$ \\ Effrosini G. Panayotopoulou, ${ }^{2}$ Eleftheria Roma-Giannikou, ${ }^{3}$ \\ Jianjun $\mathrm{Li}^{1}$ and Dionyssios N. Sgouras ${ }^{2}$ \\ ${ }^{1}$ Institute for Biological Sciences, National Research Council Canada, Ottawa, ON K1A OR6, \\ Canada \\ ${ }^{2}$ Laboratory of Medical Microbiology, Hellenic Pasteur Institute, Athens 11521, Greece \\ ${ }^{3}$ First Department of Pediatrics, Athens University School of Medicine, Athens, Greece
}

\section{INTRODUCTION}

Helicobacter pylori has been associated with chronic infection of human gastric mucosa and increased risk of gastric cancer and peptic ulcers in adults (Dunn et al., 1997). The vast majority of individuals acquire $H$. pylori in childhood. The infection often remains asymptomatic and, if left untreated, can persist into adulthood, leading to more serious pathologies. Lipopolysaccharide (LPS) is a major cell-surface component of $H$. pylori. Structural studies carried out on a number of $H$. pylori isolates (Monteiro, 2001) have resulted in a structural model of LPS, consisting of an O-chain and a core oligosaccharide

Abbreviations: CE-MS, capillary electrophoresis-mass spectrometry; $\mathrm{Cl}$, confidence interval; HRP, horseradish peroxidase; LacNAc, $\mathrm{N}$ acetyllactosamine; OD, odds ratio; PUD, peptic ulcer disease; T4SS, type IV secretion system; WCE, whole-cell indirect ELISA. that is attached to a lipid A moiety. The O-chain of the LPS in a number of $H$. pylori strains from North American and European populations expresses type $2 \mathrm{Le}^{\mathrm{x}}$ and $\mathrm{Le}^{\mathrm{y}}$ bloodgroup epitopes (Monteiro, 2001) that mimic structures present on human gastric and tumour cell surfaces and could be implicated in adverse autoimmune reactions with the host (Appelmelk et al., 1996). Recently, it has been suggested that $H$. pylori can change its Le phenotype to the type 1 pathway in vivo in order to adapt to specific conditions in the host (Pohl et al., 2009). Furthermore, it has been suggested that depending on the human host population, $\mathrm{Le}^{\mathrm{x}} / \mathrm{Le}^{\mathrm{y}}$ expression by $H$. pylori may aid the persistence of more aggressive strains (Moran, 2008).

In the present study, we evaluated LPS expression in $50 \mathrm{H}$. pylori strains from the Greek paediatric population, since children constitute a natural milieu less influenced by environmental and host-associated factors (Oleastro et al., 
2010). The isolates were screened by whole-cell indirect ELISA (WCE) using commercially available anti-Le monoclonal antibodies (mAbs) and anti- $\alpha 1,6$-glucanspecific mAbs developed in our laboratory (Harrison et al., 2011). Selected strains were further characterized in terms of carbohydrate content by using SDS-PAGE electrophoresis, immunoblotting and chemical and mass spectrometric analyses. Other virulent characteristics, such as vacA isoforms, presence and functionality of type IV secretion systems (T4SS) and the number and type of functional EPIYA-phosphorylation motifs on translocated CagA proteins, were correlated with the LPS content.

\section{METHODS}

Patients. The study included symptomatic $H$. pylori-infected children, 2-16 years old, who underwent upper endoscopy at the gastroenterology clinic of the First Department of Paediatrics of Athens University, Aghia Sophia Children's Hospital, as detailed before (Sgouras et al., 2009). The study was approved by the Hospital Scientific Committee and the Ethical Committee.

Culture. Antral mucosa biopsy specimens were processed for $H$. pylori isolation as described previously (Sgouras et al., 2009). Individual colonies were further propagated, and frozen stocks were prepared using BHI broth containing $20 \%(\mathrm{v} / \mathrm{v})$ glycerol. They were stored at $-80{ }^{\circ} \mathrm{C}$ until further analysis.

Whole-cell indirect ELISA (WCE). H. pylori strains were cultivated on plates as described previously (Logan et al., 2000). Cells were harvested and washed with $5 \mathrm{ml} 10 \mathrm{mM}$ PBS ( $\mathrm{pH}$ 7.4) per plate. Following centrifugation $\left(5000 \mathrm{~g}, 5 \mathrm{~min}, 4{ }^{\circ} \mathrm{C}\right.$ ), pellets were suspended in $25 \mathrm{ml}$ PBS to give a final concentration of $10^{8}$ cells $\mathrm{ml}^{-1}$. WCE was performed as previously described (Altman et al., 2008). Briefly, the wells of a microtitre plate were coated with $100 \mathrm{ml}$ bacterial suspension $\left(10^{8}\right.$ cells $\left.\mathrm{ml}^{-1}\right)$ overnight at $4{ }^{\circ} \mathrm{C}$. The wells were then fixed with methanol and blocked with $200 \mu$ l Milk Diluent/Blocking solution (MDB) (KPL) for $2 \mathrm{~h}$ at $37{ }^{\circ} \mathrm{C}$. Subsequently, the wells were incubated for $2 \mathrm{~h}$ at $37^{\circ} \mathrm{C}$ with either $100 \mu \mathrm{l}$ anti-Le $\mathrm{L}^{\mathrm{a}},-\mathrm{Le}^{\mathrm{b}},-\mathrm{Le}^{\mathrm{x}}$ or $-\mathrm{Le}^{\mathrm{y}} \mathrm{mAb}$ solution (Signet Laboratories) diluted 1:200 in MDB, $100 \mu \mathrm{l}$ 1C4F9 ascites (anti- $\alpha 1,6$-glucan $\mathrm{mAb}$ ) (Harrison et al., 2011) diluted 1:500 in MDB or rabbit anti-CagA antibody (Austral Biologicals) diluted $1: 100$ in MDB. This was followed by incubation with horseradish peroxidase (HRP)-conjugated anti-mouse IgG + IgM (Caltag) diluted $1: 1000$ in MDB for $1 \mathrm{~h}$ at room temperature. A corresponding secondary antibody, either HRP-anti-mouse IgG + IgM or HRP-anti-rabbit IgG (Cedarlane Laboratories) diluted 1:1000 in MDB, was added and the plates were incubated for $1 \mathrm{~h}$ at room temperature. The substrate TMB was added as described previously for indirect ELISA (Altman et al., $2005)$. $\mathrm{OD}_{450}$ values $<0.2$ were classified as negative and $\mathrm{OD}_{450}$ values $\geqslant 0.2$ were classified as positive reactions. To ensure consistency between plates, $H$. pylori strain 26695 cells were used as a positive control for $\mathrm{Le}^{\mathrm{x}}, \mathrm{Le}^{\mathrm{y}}, \alpha 1,6$-glucan and CagA. Assay results did not vary by more than $10 \%$.

Electrophoresis and immunoblotting. SDS-PAGE was performed with a mini-slab apparatus (Bio-Rad) according to the method of Laemmli (1970). LPS samples were prepared from whole cells according to a previously described method (Logan \& Trust, 1984). Equivalent amounts of sample were loaded into each lane and stained according to Tsai \& Frasch (1982). For detection of $\mathrm{Le}^{\mathrm{b}}$ in bacterial whole-cell samples, nitrocellulose membranes were blocked with $1 \%$ skimmed milk solution in $10 \mathrm{mM}$ PBS overnight at $4{ }^{\circ} \mathrm{C}$. Subsequently, membranes were incubated with anti-Le ${ }^{\mathrm{b}} \mathrm{mAb}$ solution diluted $1: 100$ in $10 \mathrm{mM}$ PBS, overnight at $4{ }^{\circ} \mathrm{C}$. The corresponding secondary antibody HRP-anti-mouse IgG + IgM diluted $1: 3000$ in $10 \mathrm{mM}$ PBS was added, and the nitrocellulose membranes were incubated for $1 \mathrm{~h}$ at room temperature. The membranes were washed three times with $10 \mathrm{mM}$ PBS between the incubation steps and developed as described previously (Altman et al., 2008) using SuperSignal West Pico Chemiluminescent substrate (Pierce), following the manufacturer's instructions.

Sugar analysis. Bacterial cells were hydrolysed using $2 \mathrm{M}$ trifluoroacetic acid at $100{ }^{\circ} \mathrm{C}$ for $16 \mathrm{~h}$ followed by reduction with sodium borohydride and subsequent acetylation as previously described (Altman et al., 2003).

CE-MS. Bacterial cells were digested with RNase and DNase (final concentration $10 \mu \mathrm{g} \mathrm{ml}^{-1}$ in $0.02 \mathrm{M}$ ammonium acetate, $\mathrm{pH}$ 7.5) for $2 \mathrm{~h}$ at $37^{\circ} \mathrm{C}$ to release LPS, and the cell pellets were delipidated with $0.1 \mathrm{M}$ sodium acetate buffer ( $\mathrm{pH}$ 4.5) (Altman et al., 2003). Samples were desalted using a centrifugal filter device (Micron-3K, molecular mass cut-off 3000; Pall Corporation). The recovered solution was analysed directly by a Crystal model $310 \mathrm{CE}$ instrument coupled to an API 3000 mass spectrometer (Li et al., 2005).

Determination of $\boldsymbol{H}$. pylori virulence characteristics. EPIYA-C motifs in CagA and the VacA vacuolating isoforms were assessed as previously described (Panayotopoulou et al., 2010; Sgouras et al., 2009). Functionality of the T4SS was assessed by testing for the presence of cagA and cagE genes, detected by PCR, and the phosphorylated CagA protein and secreted IL- 8 in the supernatant of $H$. pylori-infected AGS cells (Panayotopoulou et al., 2010; Sgouras et al., 2009).

Statistical analysis. Contingency analysis was performed, and levels of significance were verified by univariate logistic regression analysis; selection of variables was based on a likelihood ratio test, using a significance level of 0.05 for inclusion or elimination.

\section{RESULTS}

\section{LPS immunotyping of $\boldsymbol{H}$. pylori isolates}

Isolates were initially screened by WCE with anti-Le ${ }^{\mathrm{x}},-\mathrm{Le}^{\mathrm{y}}$, $-\mathrm{Le}^{\mathrm{a}}$, $-\mathrm{Le}^{\mathrm{b}}$ and $-\alpha 1,6$-glucan mAbs. The expression of $\mathrm{Le}^{\mathrm{x}}$ and/or Le ${ }^{\mathrm{y}}$ antigens was detected in 35 strains, whilst 15 strains were non-typable (Table 1). Exclusive Le $\mathrm{b}^{\mathrm{b}}$ expression was identified in three of these non-typable isolates, whilst the presence of $\mathrm{Le}^{\mathrm{b}}$ antigen was detected in 11 isolates in total, the further eight being either co-expressed with $\mathrm{Le}^{\mathrm{x}}$ and $\mathrm{Le}^{\mathrm{y}}$ (four isolates) or together with $\mathrm{Le}^{\mathrm{y}}$ (four isolates). Co-expression of $\mathrm{Le}^{\mathrm{x}}, \mathrm{Le}^{\mathrm{y}}, \mathrm{Le}^{\mathrm{b}}$ and $\mathrm{Le}^{\mathrm{a}}$ antigens was confirmed in one strain (strain 103). The expression of type 2 and/or type 1 Le antigen was confirmed in 15 CagAnegative isolates (Table 1).

The presence of $\alpha 1,6$-glucan was confirmed in 49 of the 50 strains $(98 \%)$; the expression of this antigen in one strain (strain 68) was found to be slightly below the detection limit. All non-typable $H$. pylori strains expressed $\alpha 1,6-$ glucan, regardless of their CagA status (Table 1).

\section{Structural characterization of non-typable strains}

Non-typable $H$. pylori strains were further characterized by SDS-PAGE, sugar analysis and capillary electrophoresis-mass 
Table 1. Analysis of paediatric $H$. pylori isolates from Greece with reference to expression of Lewis antigens, $\alpha 1,6$-glucan and cagA status, number of EPIYA-C motifs in CagA, functionality of T4SS and vacuolation potential of VacA

\begin{tabular}{|c|c|c|c|c|c|c|c|c|c|}
\hline Strain ${ }^{\star}$ & $\mathrm{Le}^{\mathrm{x}}$ & $\mathrm{Le}^{\mathrm{y}}$ & $\mathrm{Le}^{\mathrm{a}}$ & $\mathrm{Le}^{\mathrm{b}}$ & $\alpha 1,6$-glucan & CagA Ag & $\begin{array}{c}\text { No. EPIYA-C } \\
\text { motifs } \dagger\end{array}$ & T4SS & Vacuolation $\S$ \\
\hline 32 & - & - & - & - & 1.502 & - & 0 & - & - \\
\hline 35 & 1.318 & 1.235 & - & - & 0.338 & 1.035 & 1 & + & + \\
\hline 40 & - & - & - & - & 1.501 & - & 0 & - & - \\
\hline 42 & 1.601 & 1.009 & - & - & 0.463 & 1.244 & 1 & + & - \\
\hline 63 & 1.653 & 1.350 & - & - & 1.615 & 1.156 & 2 & + & + \\
\hline 68 & 1.045 & 1.181 & - & - & 0.194 & 1.262 & 2 & + & - \\
\hline 71 & - & - & - & - & 1.401 & - & 2 & - & - \\
\hline 72 & - & - & - & - & 1.056 & 0.766 & 1 & + & - \\
\hline 73 & 1.617 & 1.034 & - & - & 1.486 & - & 0 & - & - \\
\hline $77-2$ & - & - & - & - & 1.308 & 0.718 & 0 & + & + \\
\hline 93 & 0.623 & 1.414 & - & - & 0.597 & 1.462 & 1 & + & - \\
\hline 94 & 1.580 & 1.113 & - & - & 0.683 & 1.364 & 1 & + & + \\
\hline 97 & 0.507 & 0.956 & - & - & 1.223 & 0.399 & 1 & + & + \\
\hline 98 & - & 0.641 & - & - & 0.883 & 1.471 & 1 & + & + \\
\hline 99 & - & - & - & 0.716 & 0.260 & - & 2 & - & + \\
\hline 100 & 1.433 & 1.058 & - & - & 1.463 & 1.399 & 2 & + & + \\
\hline 101 & 0.752 & 1.451 & - & - & 1.570 & 1.508 & 2 & + & + \\
\hline 102 & - & - & - & 0.752 & 0.297 & - & 0 & - & - \\
\hline 103 & 1.022 & 0.641 & 0.312 & 0.765 & 0.913 & - & 0 & - & - \\
\hline 104 & - & 0.366 & - & - & 1.381 & - & 0 & - & - \\
\hline 105 & 0.869 & 1.171 & - & - & 1.677 & - & 0 & - & - \\
\hline $146195-8$ & - & 1.035 & - & - & 0.827 & 1.384 & 1 & + & + \\
\hline $146195-13$ & - & - & - & - & 1.688 & 0.866 & 2 & + & + \\
\hline 147181 & - & - & - & - & 1.063 & - & 0 & - & - \\
\hline 147403 & 1.701 & 1.539 & - & 0.370 & 0.463 & 1.133 & 2 & + & + \\
\hline 147570 & - & - & - & 0.406 & 0.449 & 1.146 & 1 & + & + \\
\hline 147631 & - & 0.601 & - & - & 0.395 & 1.146 & 2 & + & - \\
\hline 148455 & - & - & - & - & 0.418 & 1.185 & 1 & + & + \\
\hline 148715 & 1.769 & 1.545 & - & - & 0.441 & - & 0 & - & - \\
\hline 149489 & - & 1.457 & - & 0.824 & 1.737 & - & 0 & - & - \\
\hline 150453 & 1.633 & 1.538 & - & - & 0.489 & 0.930 & 1 & + & + \\
\hline 150934 & 1.729 & 1.611 & - & - & 1.814 & 1.074 & 2 & + & + \\
\hline 152047 & 1.040 & 1.435 & - & - & 1.602 & - & 2 & - & + \\
\hline 153085 & - & 0.556 & - & 1.142 & 0.386 & 1.063 & 1 & + & - \\
\hline 160708 & - & - & - & - & 1.810 & - & 0 & - & - \\
\hline 160729 & - & 1.544 & - & - & 1.804 & - & 0 & - & - \\
\hline 167005 & 1.597 & 1.378 & - & - & 0.508 & 1.106 & 2 & + & + \\
\hline
\end{tabular}

${ }^{\star}$ Clinical isolates belonging to the Hellenic Pasteur Institute Biobank (HPI reference number) as described before (Sgouras et al., 2009). $\dagger$ Number of EPIYA-C motifs [RS(V/A)SPEPIYATIDDLG] in CagA, assessed as described before (Sgouras et al., 2009).

¥Functional status of type IV secretion system in H. pylori strains, assessed as described previously (Panayotopoulou et al., 2010; Sgouras et al., 2009). §Vacuolation potential of VacA was assessed as described previously (Panayotopoulou et al., 2010). 
spectrometry (CE-MS) performed directly on bacterial cells. Silver staining revealed significant variations in the O-chain length of these strains. Strains 32, 40, 71, 146195-13, 147181, 147570 and 160708 appeared to contain an extended O-chain component compared with the staining pattern of $H$. pylori strain 26695, although they tested negative for the presence of $\mathrm{Le}^{\mathrm{y}}$ and $\mathrm{Le}^{\mathrm{x}}$ antigens by WCE. Sugar analysis suggested that these isolates contained reduced amounts of L-fucose compared with the cells of $H$. pylori strain 26695, which are known to carry $\mathrm{Le}^{\mathrm{x}}$ and $\mathrm{Le}^{\mathrm{y}}$ antigens (Table 2). Silver staining patterns of strains 72, 77-2 and 77-5 appeared to be almost rough and showed only very faint high-molecular-mass bands (Fig. 1). It is of note that strains 40, 146195-13 and 147181 contained increased amounts of DD-heptose compared with strain 26695, suggesting the presence of DDheptan in their LPS (Table 2) (Hiratsuka et al., 2005). H. pylori strains 32, 146195-13 and 160708 contained the highest amount of D-glucose compared to 26695 cells, suggesting the presence of a long $\alpha 1$,6-glucan chain. Non-typable $H$. pylori strains 147181, 147570 and 160708, containing different amounts of L-fucose, were selected for CE-MS analysis in the positive ion mode and the resulting spectra were compared to the CE-MS fragmentation pattern obtained for 26695 cells under the same conditions. Enzyme-treated and delipidated H. pylori cells from these strains were subjected to in-source collision-induced dissociation MS analysis (Li et al., 2005; Wang et al., 2005). This technique allows in-source fragmentation of the $\mathrm{O}$-chain polysaccharide into repeating unit components, confirming its sequence. The resulting spectra were consistent with the presence of $\mathrm{N}$-acetyllactosamine (LacNAc) repeating units, and fragment ions corresponding to LacNAc $(\mathrm{m} / z$ 366), LacNAc- $N$-acetylhexosamine $(\mathrm{m} / z 569)$ and double repeating units of LacNAcLacNAc at $\mathrm{m} / z 731$ were observed along with the fragment corresponding to the backbone core oligosaccharide $(\mathrm{m} / \mathrm{z}$ 1244) (Fig. 2).

Table 2. Chemical analysis of LPS from non-typable strains of H. pylori

Approximate molar ratios of the alditol acetate derivatives are given. Analyses were performed on whole bacterial cells. Fuc, fucose; Glc, glucose; Gal, galactose; DD-Hep, DD-heptose; DL-Hep, DL-heptose.

\begin{tabular}{|lcccccc|}
\hline Strain & Fuc & Glc & Gal & GlcNAc & DD-Hep & DL-Hep \\
\hline 32 & 0.2 & 4.0 & 2.0 & 2.4 & 1.5 & 1.0 \\
40 & 0.2 & 2.0 & 2.0 & 1.3 & 6.0 & 1.0 \\
71 & 0.2 & 2.1 & 1.2 & 1.9 & 1.5 & 1.0 \\
72 & 0.2 & 2.0 & 1.6 & 2.0 & 1.4 & 1.0 \\
$77-2$ & 0.2 & 1.0 & 0.7 & 2.2 & 1.4 & 1.0 \\
$77-5$ & 0.3 & 2.0 & 1.4 & 1.8 & 1.8 & 1.0 \\
$146195-13$ & 0.2 & 4.5 & 1.7 & 4.5 & 3.0 & 1.0 \\
147181 & 0.1 & 1.0 & 1.3 & 2.2 & 2.2 & 1.0 \\
147570 & 0.2 & 2.0 & 1.4 & 2.9 & 1.5 & 1.0 \\
160708 & 0.3 & 5.3 & 2.2 & 4.0 & 2.0 & 1.0 \\
26695 & 0.5 & 2.5 & 3.8 & 5.9 & 1.6 & 1.0 \\
& & & & & & \\
\hline
\end{tabular}

\section{Analysis of Le $e^{b}$-containing $\boldsymbol{H}$. pylori isolates}

Silver staining and Western blotting with anti-Le ${ }^{\mathrm{b}} \mathrm{mAbs}$ revealed significant variations in the O-chain length of $\mathrm{Le}^{\mathrm{b}}$ containing isolates (Fig. 3). In strains, 59, 79, 153085, 103, $108,109,147403$ and 149489 , the Le $\mathrm{b}^{\mathrm{b}}$ epitope was found to be co-expressed with $\mathrm{Le}^{\mathrm{x}}$ and/or $\mathrm{Le}^{\mathrm{y}}$ antigens $\left(8 / 11 \mathrm{Le}^{\mathrm{b}}\right.$ containing isolates). Three $\mathrm{Le}^{\mathrm{b}}$-containing strains, namely, 79, 147403 and 149489, did not react in the Western blot format but were positive for the presence of $\mathrm{Le}^{\mathrm{b}}$ antigen in WCE results. Two of these strains, 79 and 147403, had a low $\mathrm{Le}^{\mathrm{b}}$ antigen content according to WCE results (Table 1). The high-molecular-mass ladder-like pattern produced by silver staining of the third strain, 149489 , suggested the presence of a long $\alpha 1,6$-glucan chain, in addition to $\mathrm{Le}^{\mathrm{y}}$ and $\mathrm{Le}^{\mathrm{b}}$ antigens, as corroborated by WCE data (Fig. 3a). Three $\mathrm{Le}^{\mathrm{b}}$-positive but $\mathrm{Le}^{\mathrm{x}}$ - and $\mathrm{Le}^{\mathrm{y}}$-negative strains $(99,102$ and 147570) reacted in the Western blot format with anti-Le ${ }^{\mathrm{b}}$ $\mathrm{mAbs}$ and showed very diverse $\mathrm{O}$-chain profiles capped by $\mathrm{Le}^{\mathrm{b}}$ structures comprising a long O-chain in strain 99, an intermediate $\mathrm{O}$-chain in strain 102 and a short O-chain in strain 147570 (Fig. 3b). Due to the limited number of $\mathrm{Le}^{\mathrm{b}}$-expressing cases, we were not able to produce any meaningful statistical analysis relating to potential correlation with data from cases of more severe histopathology or greater bacterial density.

\section{Co-expression of $\mathrm{Le}^{\mathrm{y}}$ and $\mathrm{Le}^{\mathrm{x}}$ in more virulent strains of $\boldsymbol{H}$. pylori}

Co-expression of $\mathrm{Le}^{\mathrm{x}}$ and $\mathrm{Le}^{\mathrm{y}}$ antigens was observed in $H$. pylori strains possessing vacuolating-inducing VacA forms [16/50, $32 \%$, odds ratio (OD): 3.2, $95 \%$ confidence interval (CI): 1.004-10.203] or functional T4SS $(17 / 50,34 \%$, OR: 3.886, $95 \%$ CI: $1.191-12.681)$, or both (14/50, $28 \%$, OR: 3.800 , 95 \% CI: 1.159-12.456). However, no correlation was observed between the LPS content and the number of EPIYA phosphorylation motifs in the CagA protein (data not shown). Furthermore, no association was observed between the severity of histopathological findings (for chronic gastritis, $P=0.562$, Pearson's chi-squared $=1.154$; for chronic inflammatory infiltration, $P=0.427$, Pearson's chi-squared $=1.704$ ) or the levels of bacterial colonization in the antrum $(P=0.777$, Pearson's chi-squared $=0.506)$ and the distribution of $\mathrm{Le}^{\mathrm{x}}$ and $\mathrm{Le}^{\mathrm{y}}$ antigens (data not shown). Finally, no statistical difference was observed in terms of histopathological features, especially bacterial density, between strains expressing type 1 (or nontypable) and type 2 Lewis antigens.

\section{DISCUSSION}

This study examined the expression of Le antigens and al,6-glucan in $50 \mathrm{H}$. pylori isolates from symptomatic Greek children. While the majority of the strains examined expressed type $2 \mathrm{Le}^{\mathrm{x}}$ and/or $\mathrm{Le}^{\mathrm{y}}$ antigens (70\%), we showed that, unlike $H$. pylori strains isolated from adult population, paediatric isolates have the tendency for expression of the type $1 \mathrm{Le}^{\mathrm{b}}$ blood group antigen $(22 \%)$. 


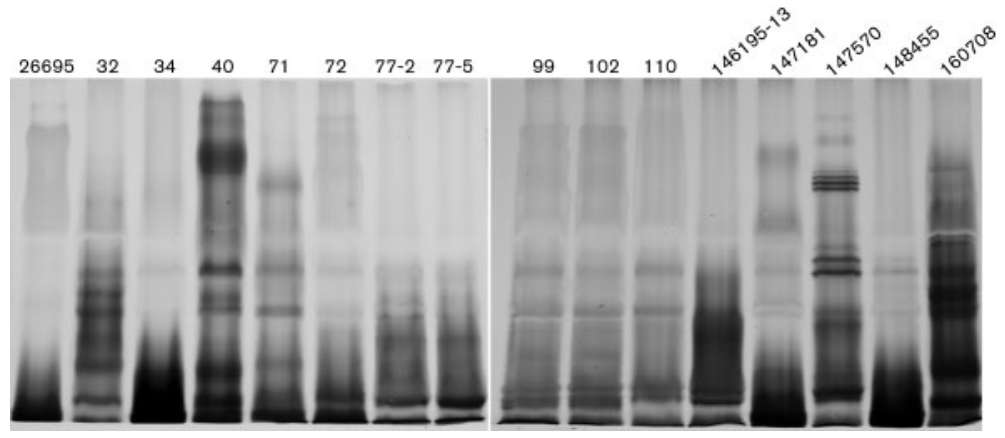

Fig. 1. Silver-stained SDS-PAGE (12.5\% acrylamide) of proteinase K-treated whole cells of $H$. pylori.

In accordance with our previous findings (Altman et al., $2008)$, in the majority of cases, the Le $\mathrm{L}^{\mathrm{b}}$ antigen was either co-expressed with type $2 \mathrm{Le}^{\mathrm{x}}$ and $\mathrm{Le}^{\mathrm{y}}$ antigens or just with $\mathrm{Le}^{\mathrm{y}}$. Three $\mathrm{Le}^{\mathrm{b}}$-positive isolates were non-typable and produced LPS O-chains composed of LacNAc units capped by $\mathrm{Le}^{\mathrm{b}}$ antigens. Recent studies have demonstrated that $H$. pylori populations expressing $\mathrm{Le}^{\mathrm{b}}$ were more efficient than non-Le ${ }^{\mathrm{b}}$ expressers in colonizing Le $\mathrm{e}^{\mathrm{b}}$-transgenic mice (Pohl et al., 2009). In addition, it was shown that the $j h p 562$ gene, encoding $\beta$-1,3-galactosyltransferase, which is involved in (a)

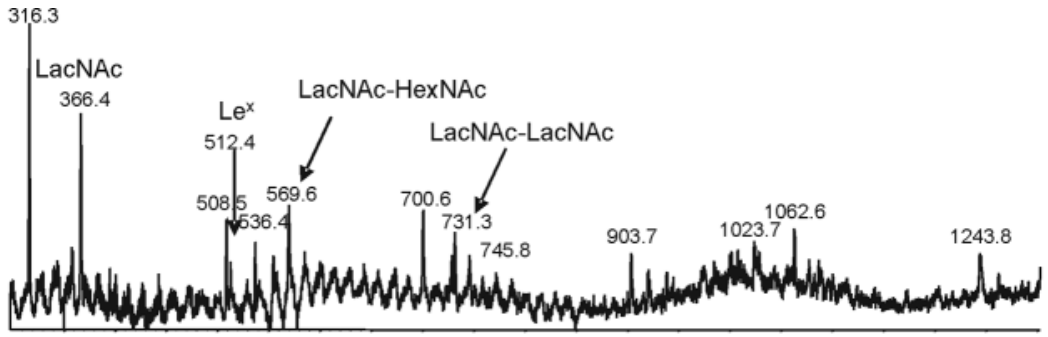

(b)

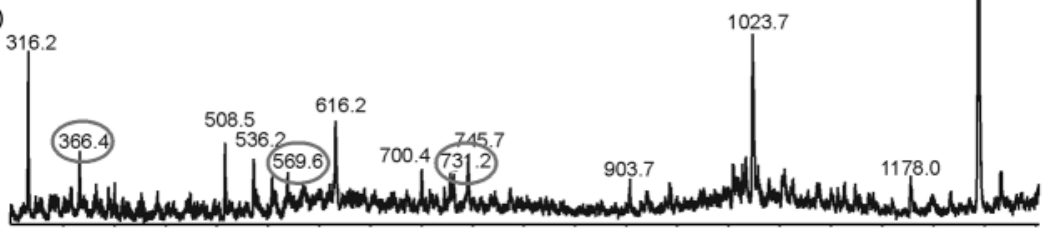

(c) 316.2

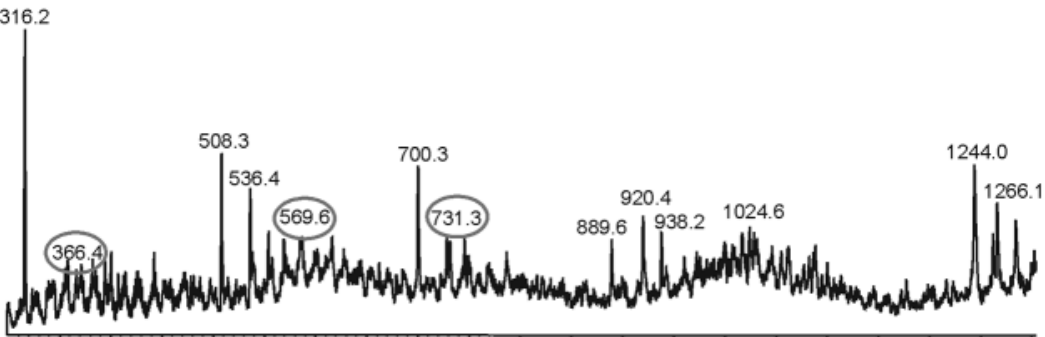

(d)

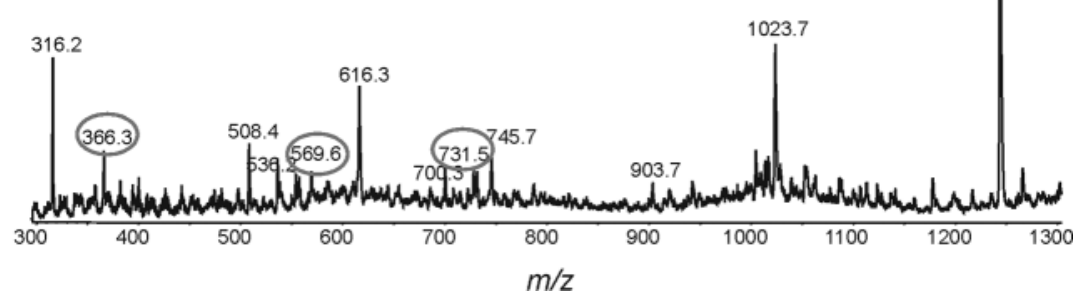

Fig. 2. CE-MS analysis of $H$. pylori cells in the positive ion mode using orifice voltage of 380 V. (a) Strain 26695 (control), (b) strain 147181, (c) strain 160708, (d) strain 147570. 

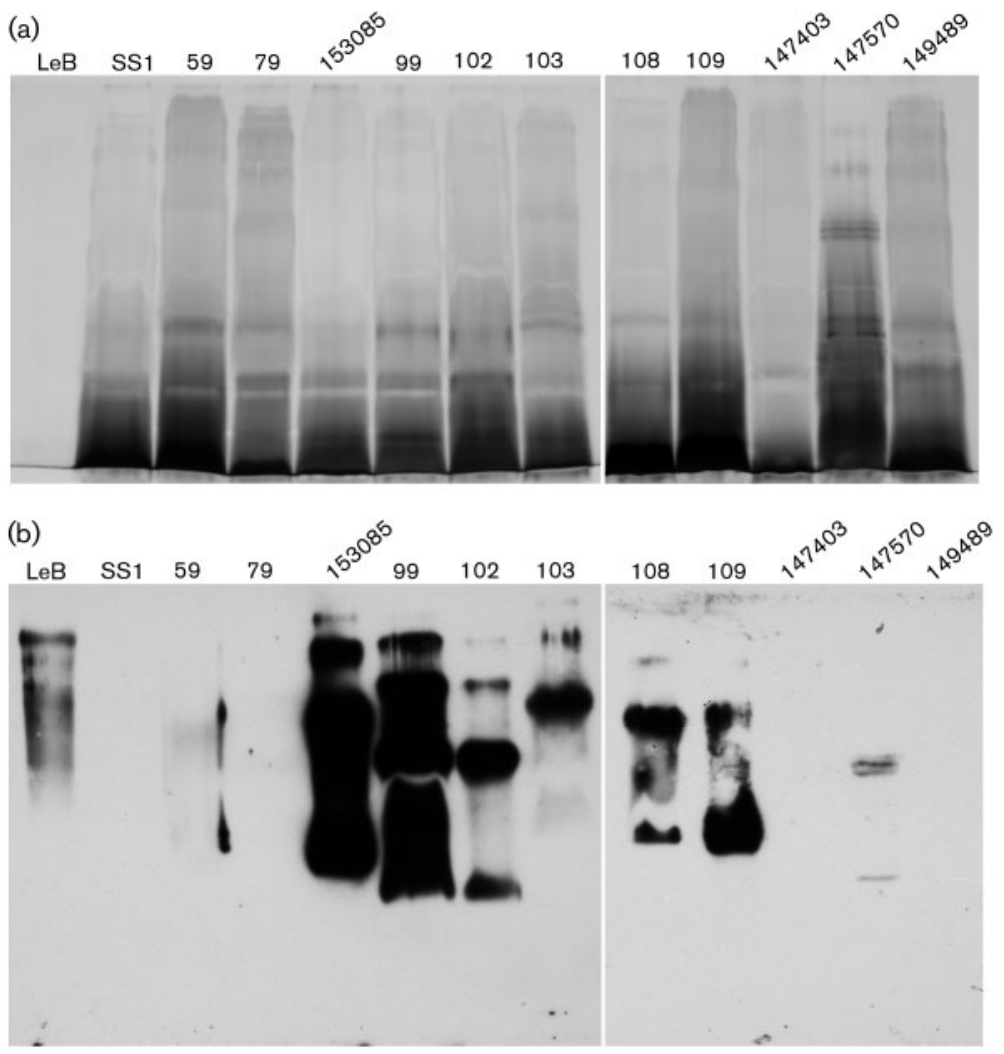

Fig. 3. Silver-stained SDS-PAGE (12.5\% acrylamide) (a) and immunoblot (b) of proteinase K-treated whole cells of $H$. pylori Le ${ }^{\mathrm{b}}$ containing isolates. $H$. pylori strain SS1, negative control; LeB, Le ${ }^{b}$-hexasaccharideBSA, positive control. the biosynthesis of type $1 \mathrm{Le}^{\mathrm{a}}$ and $\mathrm{Le}^{\mathrm{b}}$ antigens, is associated with peptic ulcer disease (PUD) in Portuguese children (Oleastro et al., 2010). Furthermore, a previous study of Taiwanese isolates of $H$. pylori provided evidence that the intensity of gastric $\mathrm{Le}^{\mathrm{b}}$ and $\mathrm{Le}^{\mathrm{x}}$ determinants could be correlated with bacterial density of $H$. pylori colonization in the stomach (Sheu et al., 2003).

We did not find any correlation between histopathological findings or referral symptoms in children and the presence of the Le $e^{b}$ antigen. However, most of the strains examined in this study were from symptomatic children presenting with epigastralgia, vomiting and occasional oesophagitis and only two isolates were from children with PUD. Expression of $\mathrm{Le}^{\mathrm{b}}$ in combination with $\mathrm{Le}^{\mathrm{y}}$ was confirmed in both strains associated with PUD, in line with previous observations (Oleastro et al., 2010). Four out of six $H$. pylori isolates from children experiencing vomiting were non-typable and one out of the six strains expressed Le ${ }^{b}$ exclusively. It has been previously shown that a higher intensity of gastric $\mathrm{Le}^{\mathrm{b}}$ expression over the corpus could be correlated with an increased risk of ulcers in children (Yang et al., 2008). More studies are needed to confirm the association between the expression of the $\mathrm{Le}^{\mathrm{b}}$ phenotype and $H$. pylori infection in children.

It has been well established that $80 \%$ of $\operatorname{cag} A$-positive $H$. pylori strains express $\mathrm{Le}^{\mathrm{x}}$ or $\mathrm{Le}^{\mathrm{y}}$ and more than half of them express both (Heneghan et al., 2000; Wirth et al., 1996). Previous studies also suggested that the expression of type
2 Le antigens by $\operatorname{cag} A$-positive isolates could counterbalance their proinflammatory effects (Wirth et al., 1996). In a more recent study (Yahav et al., 2000), it was shown that anti-CagA-seropositive $H$. pylori-infected children have a significantly higher incidence of persistent infection and harder-to-treat gastroduodenal disease. In contrast, in this study 15 of 35 strains expressing type 2 and/or type 1 Le antigens $(30 \%)$ were CagA-negative. Similar findings were previously reported by Gold et al. (2001) who observed the presence of marked gastritis in $27 \%$ of cagA-negative $H$. pylori strains isolated from North American children. As previously suggested, loss of cagPAI and the prevalence of cagA- and T4SS-deficient isolates in children could be evolutionary and may offer a selective advantage during early colonization (Sgouras et al., 2009).

Two pairs of strains examined in the present study, 146195-8 and 146195-13, and 105 and 160708, were isolated from children who underwent upper endoscopy, twice and at different ages. In both instances, the expression of Le antigens was associated with $H$. pylori isolates from younger children and disappeared with age. These results are consistent with previous observations that changes in gastric Le expression are different between adults and children and that the H. pylori-infected adults, but not the children, had a lower Le $\mathrm{e}^{\mathrm{b}}$ intensity over the antrum but higher intensity over the corpus than non-infected ones, as shown by immunohistochemical staining of gastric biopsies (Yang et al., 2008). Furthermore, H. pylori-infected adults expressed higher 
levels of $\mathrm{Le}^{\mathrm{b}}$ and had a higher bacterial density in both antrum and corpus as compared with those with weak $\mathrm{Le}^{\mathrm{b}}$ intensity (Yang et al., 2008). Two of the examined strains, 77-2 and 77-5, represented different isolates from the same patient; both strains exhibited similar expression levels of $\alpha 1,6$-glucan as determined by WCE and had similar LPS patterns as determined by silver staining, although they have been shown to express CagA protein with different numbers of EPIYA motifs (Sgouras et al., 2009).

An unusually high frequency of non-typable isolates (30\%) was also observed. It was previously suggested that the lack of Le expression in cagA-positive isolates is uncommon (Wirth et al., 1996). However, in our study, six out of 15 non-typable strains $(40 \%)$ were CagA-positive by WCE. Selected non-typable strains were subjected to further characterization by composition and CE-MS analyses, performed directly on bacterial cells. The results confirmed the presence of mainly LacNAc-containing structures in these isolates and low fucosylation levels. The prognostic significance of these findings remains to be established.

Another interesting feature of paediatric isolates of $H$. pylori is the overwhelming presence of $\alpha 1,6$-glucan. We recently reported that $44.7 \%$ of Chilean $H$. pylori isolates expressed $\alpha 1,6$-glucan, a polymeric component of the outer core region of LPS (Harrison et al., 2011). Co-expression of $\alpha 1,6$-glucan with $\mathrm{Le}^{\mathrm{x}}$ and/or $\mathrm{Le}^{\mathrm{y}}$ antigens in the majority of $H$. pylori strains examined suggests the importance of this LPS component in the pathogenesis of H. pylori. We have previously shown that $\alpha 1,6$-glucan affects the colonizing ability of $H$. pylori and is required for initial colonization of the murine stomach (Altman et al., 2008). In this context, the presence of $\alpha 1,6$-glucan in $H$. pylori strains isolated from children could be considered another important phenotypic characteristic facilitating successful colonization and contributing to the antigenic diversity of $H$. pylori LPS.

\section{REFERENCES}

Altman, E., Smirnova, N., Li, J., Aubry, A. \& Logan, S. M. (2003). Occurrence of a nontypable Helicobacter pylori strain lacking Lewis blood group O antigens and DD-heptoglycan: evidence for the role of the core $\alpha 1,6$-glucan chain in colonization. Glycobiology 13, 777-783.

Altman, E., Harrison, B. A., Hirama, T., Chandan, V., To, R. \& MacKenzie, R. (2005). Characterization of murine monoclonal antibodies against Helicobacter pylori lipopolysaccharide specific for $\mathrm{Le}^{\mathrm{x}}$ and Le ${ }^{\mathrm{y}}$ blood group determinants. Biochem Cell Biol 83, 589-596.

Altman, E., Fernández, H., Chandan, V., Harrison, B. A., Schuster, M. W., Rademacher, L. O. \& Toledo, C. (2008). Analysis of Helicobacter pylori isolates from Chile: occurrence of selective type 1 Lewis b antigen expression in lipopolysaccharide. J Med Microbiol 57, 585-591.

Appelmelk, B. J., Simoons-Smit, I., Negrini, R., Moran, A. P., Aspinall, G. O., Forte, J. G., De Vries, T., Quan, H., Verboom, T. \& other authors (1996). Potential role of molecular mimicry between Helicobacter pylori lipopolysaccharide and host Lewis blood group antigens in autoimmunity. Infect Immun 64, 2031-2040.
Dunn, B. E., Cohen, H. \& Blaser, M. J. (1997). Helicobacter pylori. Clin Microbiol Rev 10, 720-741.

Gold, B. D., van Doorn, L. J., Guarner, J., Owens, M., Pierce-Smith, D., Song, Q., Hutwagner, L., Sherman, P. M., de Mola, O. L. \& Czinn, S. J. (2001). Genotypic, clinical, and demographic characteristics of children infected with Helicobacter pylori. J Clin Microbiol 39, 1348-1352.

Harrison, B. A., Fernández, H., Chandan, V., Schuster, M. W., Rademacher, L. O., Toledo, C., Li, J. \& Altman, E. (2011). Characterization and functional activity of murine monoclonal antibodies specific for $\alpha 1,6$-glucan chain of Helicobacter pylori lipopolysaccharide. Helicobacter 16, 459-467.

Heneghan, M. A., McCarthy, C. F. \& Moran, A. P. (2000). Relationship of blood group determinants on Helicobacter pylori lipopolysaccharide with host Lewis phenotype and inflammatory response. Infect Immun 68, 937-941.

Hiratsuka, K., Logan, S. M., Conlan, J. W., Chandan, V., Aubry, A., Smirnova, N., Ulrichsen, H., Chan, K. H., Griffith, D. W. \& other authors (2005). Identification of a D-glycero-D-manno-heptosyltransferase gene from Helicobacter pylori. J Bacteriol 187, 5156-5165.

Laemmli, U. K. (1970). Cleavage of structural proteins during the assembly of the head of bacteriophage T4. Nature 227, 680-685.

Li, J., Wang, Z. \& Altman, E. (2005). In-source fragmentation and analysis of polysaccharides by capillary electrophoresis/mass spectrometry. Rapid Commun Mass Spectrom 19, 1305-1314.

Logan, S. M. \& Trust, T. J. (1984). Structural and antigenic heterogeneity of lipopolysaccharides of Campylobacter jejuni and Campylobacter coli. Infect Immun 45, 210-216.

Logan, S. M., Conlan, J. W., Monteiro, M. A., Wakarchuk, W. W. \& Altman, E. (2000). Functional genomics of Helicobacter pylori: identification of a beta-1,4 galactosyltransferase and generation of mutants with altered lipopolysaccharide. Mol Microbiol 35, 11561167.

Monteiro, M. A. (2001). Helicobacter pylori: a wolf in sheep's clothing: the glycotype families of Helicobacter pylori lipopolysaccharides expressing histo-blood groups: structure, biosynthesis, and role in pathogenesis. Adv Carbohydr Chem Biochem 57, 99-158.

Moran, A. P. (2008). Relevance of fucosylation and Lewis antigen expression in the bacterial gastroduodenal pathogen Helicobacter pylori. Carbohydr Res 343, 1952-1965.

Oleastro, M., Santos, A., Cordeiro, R., Nunes, B., Mégraud, F. \& Ménard, A. (2010). Clinical relevance and diversity of two homologous genes encoding glycosyltransferases in Helicobacter pylori. J Clin Microbiol 48, 2885-2891.

Panayotopoulou, E. G., Sgouras, D. N., Papadakos, K. S., Petraki, K., Breurec, S., Michopoulos, S., Mantzaris, G., Papatheodoridis, G., Mentis, A. \& Archimandritis, A. (2010). CagA and VacA polymorphisms are associated with distinct pathological features in Helicobacter pylori-infected adults with peptic ulcer and non-peptic ulcer disease. J Clin Microbiol 48, 2237-2239.

Pohl, M. A., Romero-Gallo, J., Guruge, J. L., Tse, D. B., Gordon, J. I. \& Blaser, M. J. (2009). Host-dependent Lewis (Le) antigen expression in Helicobacter pylori cells recovered from Leb-transgenic mice. J Exp Med 206, 3061-3072.

Sgouras, D. N., Panayotopoulou, E. G., Papadakos, K., MartinezGonzalez, B., Roumbani, A., Panayiotou, J., vanVliet-Constantinidou, C., Mentis, A. F. \& Roma-Giannikou, E. (2009). CagA and VacA polymorphisms do not correlate with severity of histopathological lesions in Helicobacter pylori-infected Greek children. J Clin Microbiol 47, 2426-2434.

Sheu, B. S., Sheu, S. M., Yang, H. B., Huang, A. H. \& Wu, J. J. (2003). Host gastric Lewis expression determines the bacterial density of Helicobacter pylori in babA2 genopositive infection. Gut 52, 927-932. 
Tsai, C. M. \& Frasch, C. E. (1982). A sensitive silver stain for detecting lipopolysaccharides in polyacrylamide gels. Anal Biochem 119, 115119.

Wang, Z., Vinogradov, E., Larocque, S., Harrison, B. A., Li, J. \& Altman, E. (2005). Structural and serological characterization of the O-chain polysaccharide of Aeromonas salmonicida strains A449, 80204 and 80204-1. Carbohydr Res 340, 693-700.

Wirth, H. P., Yang, M., Karita, M. \& Blaser, M. J. (1996). Expression of the human cell surface glycoconjugates Lewis $\mathrm{x}$ and Lewis $\mathrm{y}$ by
Helicobacter pylori isolates is related to cagA status. Infect Immun 64, 4598-4605.

Yahav, J., Fradkin, A., Weisselberg, B., Diver-Haver, A., Shmuely, H. \& Jonas, A. (2000). Relevance of CagA positivity to clinical course of Helicobacter pylori infection in children. J Clin Microbiol 38, 3534-3537.

Yang, Y. J., Wu, J. J., Sheu, B. S., Chen, C. R., Lu, C. C. \& Yang, H. B. (2008). Helicobacter pylori infection can change the intensity of gastric Lewis antigen expressions differently between adults and children. J Biomed Sci 15, 29-36. 